\title{
Decoding of novel missense TSC2 gene variants using in-silico methods
}

\author{
Shruthi Sudarshan ${ }^{1 *}$ (D), Manoj Kumar ${ }^{2}$, Punit Kaur ${ }^{2}$, Atin Kumar ${ }^{3}$, Sethuraman G. ${ }^{4}$, Savita Sapra', Sheffali Gulati ${ }^{5}$,
} Neerja Gupta', Madhulika Kabra' and Madhumita Roy Chowdhury

\begin{abstract}
Background: Mutations in TSC1 or TSC2 gene cause tuberous sclerosis complex (TSC), an autosomal dominant disorder characterized by the formation of non-malignant hamartomas in multiple vital organs. TSC1 and TSC2 gene products form TSC heterodimer that senses specific cell growth conditions to control mTORC1 signalling.

Methods: In the present study 98 TSC patients were tested for variants in TSC1 and TSC2 genes and 14 novel missense variations were identified. The pathogenecity of these novel variations was determined by applying different bioinformatics tools involving computer aided protein modeling.

Results: Protein modelling could be done only for ten variants which were within the functional part of the protein. Homology modeling is the most reliable method for structure prediction of a protein. Since no sequence homology structure was available for the tuberin protein, three dimensional structure was modeled by a combination of homology modeling and the predictive fold recognition and threading method using Phyre2 threading server. The best template structures for model building of the TSC1 interacting domain, tuberin domain and GAP domain are the crystal structures of clathrin adaptor core protein, Rap1GAP catalytic domain and Ser/Thr kinase Tor protein respectively.
\end{abstract}

Conclusions: In this study, an attempt has been made to assess the impact of each novel missense variant based on their TSC1-TSC2 hydrophobic interactions and its effect on protein function.

Keywords: Tuberous sclerosis complex (TSC), Multiplex ligation dependent probe amplification (MLPA), Central nervous system (CNS), GAP (GTPase-activating protein), HGMD (human gene mutation database)

\section{Background}

Tuberous Sclerosis Complex (TSC) is a rare genetic multiorgan disorder that forms non-malignant hamartomas in brain, retina, lungs, kidneys, heart and skin. The central nervous system (CNS), skin and the renal system are most commonly affected in TSC patients. The neurological manifestations include onset of epilepsy at an infantile age, autistic features, cognitive and behavioural problems ranging from mild to severe due to the presence of structural brain abnormalities like cortical tubers, subependymal nodules (SEN), white matter lesions (WML), and subependymal giant cell astrocytomas (SEGAs) [1]. TSC is transmitted in an autosomal dominant pattern of inheritance and occurs due to

\footnotetext{
* Correspondence: shruthisudarshan@yahoo.co.in

'Division of Genetics, Department of Pediatrics, AlIMS, New Delhi, India Full list of author information is available at the end of the article
}

mutations in one of two genes, TSCland TSC2. TSC1 gene consists of 23 exons, of which 21 encode hamartin $(130 \mathrm{kDa})$, with an $8.5 \mathrm{~kb}$ mRNA including a $4.5 \mathrm{~kb} 3$ 'untranslated region. The gene occupies a genomic extent of $55 \mathrm{~kb}$ on $9 \mathrm{q} 34$. The TSC 2 gene consists of 42 exons, of which 41 encode tuberin $(200 \mathrm{kDa})$, with a $5.5 \mathrm{~kb}$ mRNA and relatively short $5^{\prime}$ and $3^{\prime}$ UTRs [2]. Both these proteins function as tumour suppressors by mediating controlled multiple cellular pathways in mammalian cells. Structurally tuberin consists of 1807 amino acids with 188 residues at carboxy terminal region having homology to the catalytic domain of Rap1/Rab5 GTPase activating proteins [3]. Tuberin plays a critical role in the regulation of cell cycle progression, differentiation and development.

Tuberin and hamartin bind together to form a GTPase activating complex (TSC1/2 complex) that plays a critical role in the regulation of protein synthesis,

(c) The Author(s). 2019 Open Access This article is distributed under the terms of the Creative Commons Attribution 4.0 International License (http://creativecommons.org/licenses/by/4.0/), which permits unrestricted use, distribution, and reproduction in any medium, provided you give appropriate credit to the original author(s) and the source, provide a link to the Creative Commons license, and indicate if changes were made. The Creative Commons Public Domain Dedication waiver (http://creativecommons.org/publicdomain/zero/1.0/) applies to the data made available in this article, unless otherwise stated. 
controlling cell growth and size [4]. These two proteins do not share any homology and with other proteins also very limited homology has been observed. The only evident homology that has been detected is the putative functional domain at the C-terminal of TSC2 to the GAP (GTPase-activating protein) domain of Rap1-Gap [5]. The TSC1/2 exhibits GAP activity for the small GTPase, RHEB (Ras homolog enriched in brain), converting it from the active GTP bound form to the inactive state GDP bound [6]. GTP-bound RHEB promotes the kinase activity of mechanistic target of rapamycin complex1 (mTORC1), which phosphorylates a variety of downstream targets, including ribosomal S6 kinase 1 (S6K1) and eLF-4E binding protein 1 (4E-BP1) to stimulate the anabolic process like protein translation and lipid synthesis and inhibit the catabolic process such as autophagy [7]. Besides, any missense mutation in either of the TSC1/2 are likely to disrupt the complex formation thereby causing the disease. TSC causing mutations are identified in about 75 to $90 \%$ of the patients while in approximately10 to $15 \%$, no pathogenic variant is identified [8]. In this study we have made an effort to ascertain the pathogenecity of novel missense variants identified in TSC patients using different bioinformatics tools and computer aided protein modeling.

\section{Methods}

\section{Patient enrollment}

Over a period of 5 years from 2012 to 2017, 98 patients from the age group of 0 to 16 years were enrolled from the Pediatric Neurology Clinic and Genetics Clinic of All India Institute od Medical Sciences, New Delhi. The study was approved by the institutional ethical committee and written informed consent was taken from the parents of those enrolled. Detailed clinical proforma was filled for each patients.. The diagnosis of TSC was based upon the updated clinical criteria [9] which exhibits definite and possible diagnosis based on the presence of major and minor clinical features.

\section{Molecular analysis for TSC}

Sanger sequencing was performed on genomic DNA. Sequence analysis was performed by using ChromasPro software, NCBI BLAST and UCSC browser BLAT. For data analysis, all the variations were checked with the available human genetic variation databases online at HGMD (Human gene mutation database, http://www. hgmd.org/), dbSNP database, 1000 Genome Projects database, Ensembl browser and LOVD (http://chromium.liacs. nl/LOVD2).

\section{Computational assessment tools for novel missense variants}

To predict the functional impact of novel missense variants, in silico prediction softwares like Polyphen [10],
SIFT [11], and Mutation taster [12] were used and the splice site variant was checked with Human Splicing Finder version 2 and an improved splice site predictor tool BDGP (Berkeley Drosophila Genome Project) and Sroogle were also used to check whether this particular nucleotide change is likely to create a splice-site or not.

\section{Protein modelling}

Three dimensional (3D) structural details of the protein help to understand their structure-function relationship at the atomic level. In the absence of an experimentally determined structure, homology modelling remains a reliable tool for the structure prediction of proteins. However the homology modelling of either domain of this protein is not possible because of the absence of any homologous structure. Hence, the second most common method to predict the 3D structure of protein, i.e. fold recognition was adopted. Threading has been used to develop the 3D model structure of the three domains (TSC1 interacting domain, tuberin domain and GAP domain) using threading server Phyre2 [13]. Model for TSC1 interacting domain, tuberin domain and GAP domain of human TSC2 have been generated by combining the molecular modeling program Discovery Studio v (22) and Phyre2 server using crystal structure of clathrin adaptor core protein (PDB: 2VGL) [14], Rap1-GAP domain (PDB: 1SRQ) [15] and cryo-electron microscopy structure of Ser/Thr kinase Tor protein (PDB: 5FVM) [16] respectively. Each of these models has been energy minimized to relax the structure and remove steric constraints and verified for stereochemical quality. Model for individual variants have been built with the help of build mutant program using the optimized model structure of wild type protein. In order to analyse the structural implications of the mutant protein, the mutant protein was subjected to $20 \mathrm{~ns}$ molecular dynamics simulations using Gromacs Software [17].

\section{Results}

Identification and characterization of TSC1/TSC2 variants

Among the total 98 TSC cases enrolled, 74 were sporadic and 24 were familial. MLPA testing for both TSC2 gene and TSC1 gene was done in all and results showed the presence of large genomic rearrangements in 15 out of 98 cases enrolled. Mutation analysis of the coding exons and the intron/exon junctions by Sanger sequencing was carried out in the remaining 85 TSC cases.

Among the total 85 TSC cases, 71 different variants were identified in either TSC1 and TSC2 gene in 78 cases, while no mutation was found in 7 cases. Out of 71 variants, 64 were found in TSC2 gene while remaining 7 of these in TSC1 gene. The 7 TSC1 variants included 3 nonsense, 1 splice-site, 1 small deletion and 2 small insertion variants, while the 64 TSC2 variants included 23 
missense, 17 splice-site, 11 nonsense, 9 small deletion, and 4 small insertion variants.

Out of the total 23 missense variants, 9 missense variants are already reported as disease causing variants while the remaining 14 likely pathogenic variants were first checked in parents and siblings and in the multiple databases which included LOVD, Ensemble, HGMD, 1000 genome and also in different repository databases of Indian population as well as from other countries before these variants were marked as novel. List of all 14 novel missense variants in TSC2 gene is shown in Table 1.

Of the 14 observed novel missense variants found in tuberin protein, 10 observed variants (Tyr194Phe, Val299Glu, Leu353Pro, Lys506Glu, Glu546Lys, Leu612Pro, Cys644Gly, Leu717Pro, Pro1497Leu and Val1673Ala) lie in the defined regions of $\mathrm{N}$ terminal TSC1 interacting region (domain 1), tuberin (domain 2) and GAP (domain 3) respectively and only those could be modelled to study their effect on the structure of the protein. Figure 1 represents domain structure of TSC2 protein and also specifies the position of each novel missense variants in the respective domains.

\section{Structural characterization for novel missense variants identified in TSC2 gene}

Human TSC2 protein comprises of 1807 residues, and acts as a tumour suppressor in complex with TSC1. Three regions, $\mathrm{N}$ terminal TSC1 interacting region (residues 55 to 469), tuberin type domain (residues 555 to 903) and GTPase activator (GAP) domain (residues 1562 to 1748) are distinct on the basis of sequence similarity search with protein domain families. The pathogenecity of the various variants observed was assessed by comparing the modelled three-dimensional structures of the wild type protein with the respective individual mutant structures. The variants were categorized based on their presence in specific domain regions.

\section{Domain 1 (N terminal TSC1 interacting region): the $N$ terminal TSC1 interacting region in tuberin protein comprises of alpha-alpha superhelix domain}

This domain is stabilized by hydrophobic interactions between each helix.

Four variants were found in this domain (p.Tyr194Phe, p.Leu353Pro, p.Arg454Lys, and p.Val299Glu. Protein modeling was done for Tyr194Phe, Val299Glu, and Leu353Pro (Fig. 2). For p.Arg454Lys, no homology protein sequence could be identified to generate model structure.

The TSC1 interacting region in tuberin protein (residues 55 to 469) comprises an alpha-alpha superhelix domain encompasses the variants involving residues Tyr194, Val299 and Leu353. The hydrophobic residue Val299 located in the middle of the long helix (Fig. 3a) forms van der Waals contacts with three other hydrophobic residues Ile273, Met280 and Phe323 occurring on neighbouring helices. These interactions contribute to stabilize the helix bundle of alpha-alpha superhelix domain. The substitution of hydrophobic residue Val299 by a highly polar and negatively charged residue Glu299 (Fig. 3) not only disrupts the hydrophobic interactions between helices, but also changes the non-polar environment of the buried area in the domain into negatively charged polar environment (Fig. 3b). This might destabilize the alpha-alpha helix bundle and affect the interaction with TSC1.

In case of L353P variant, the hydrophobic residue Leu353 lies at the beginning of the helix and is stabilized through interactions with the neighbouring hydrophobic residue Ile365. This hydrophobic interaction contributes

Table 1 List of 14 novel missense TSC2 mutations

\begin{tabular}{|c|c|c|c|c|c|c|}
\hline S.No. & Patient ID & Inheritance & TSC2 Exon Number & Mutation Site & Amino acid alteration & Domain-wise distribution in tuberin \\
\hline 1 & TS-116 & Sporadic & 20 & c. $2150 \mathrm{~T}>\mathrm{C}$ & p.Leu717Pro & Domain 2 \\
\hline 2 & TS-7 & Familial & 34 & c. $4490 C>T$ & p.Pro1497Leu & Domain 3 \\
\hline 3 & TS-9 & Sporadic & 6 & c. $581 \mathrm{~A}>\mathrm{T}$ & p.Tyr194Phe & Domain 1 \\
\hline 4 & TS-97 & Sporadic & 18 & c.1930T> G & p.Cys644Gly & Domain 2 \\
\hline 5 & TS-59 & Sporadic & 14 & c. $1516 \mathrm{~A}>\mathrm{G}$ & p.Lys506Glu & Domain 2 \\
\hline 6 & TS-101 & Sporadic & 38 & c. $4859 \mathrm{~A}>\mathrm{C}$ & p.His1620Pro & Domain 3 \\
\hline 7 & TS-112 & Familial & 39 & c. $5018 \mathrm{~T}>\mathrm{C}$ & p.Val1673Ala & Domain 3 \\
\hline 8 & TS-67 & Sporadic & 11 & c.1058 T > C & p.Leu353Pro & Domain 1 \\
\hline 9 & TS-35 & Sporadic & 16 & c. $1835 \mathrm{~T}>\mathrm{C}$ & p.Leu612Pro & Domain 2 \\
\hline 10 & TS-54 & Sporadic & 16 & c. $1636 \mathrm{G}>\mathrm{A}$ & p.Glu546Lys & Domain 2 \\
\hline 11 & TS-42 & Familial & 13 & c. $1361 G>A$ & p.Arg454Lys & Domain 1 \\
\hline 12 & TS-43 & Sporadic & 31 & c. $3713 C>T$ & p.Ala1238Val & Domain 3 \\
\hline 13 & TS-48 & Sporadic & 10 & c.896 T > A & p.Val299Glu & Domain 1 \\
\hline 14 & TS-32 & Sporadic & 31 & c. $3715 G>A$ & p.Glu1230Lys & Domain 3 \\
\hline
\end{tabular}




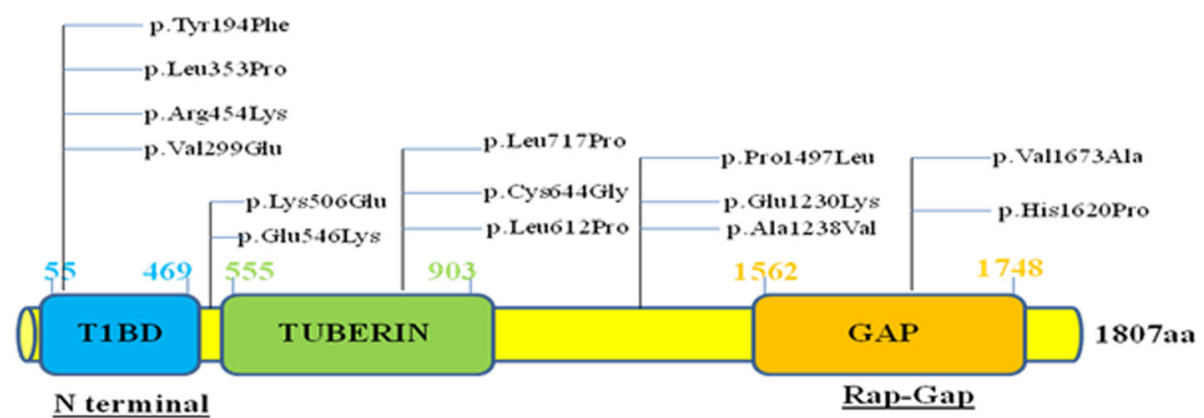

Fig. 1 Schematic representation of 13 novel TSC2 missense variants identified in respective domains of TSC2 protein

in keeping the motif intact (Fig. 3c). Substitution of Leu353 in mutant protein by the imino group containing Pro353 (Fig. 3) disrupts the helix at this position and substantially weakens the hydrophobic interaction with respect to wild type protein (Fig. 3d). As a result it most likely adversely affects the functioning of this protein.

In the wild structure, the hydroxyl group of Tyr194 side chain interacts with the side chain of Glu158 through hydrogen bonds which contribute to stabilize the helix interfaces (Fig. 3e). The substitute Phe in case of Tyr194Phe mutant though like Tyr is an aromatic residue but lacks the hydroxyl group present on the Tyr side chain. This results in the loss of the hydrogen bond which in turn weakens the interaction between two helices and decreases the overall stability of the domain.

Clinical assessment The detailed clinical assessment for all 4 TSC patients showed presence of early onset of seizures and presence of brain lesions in the form of cortical tubers, SENs and WMLs. The dermatological features included only presence of ash leaf spots as all patients were below 5 years of age.

\section{Domain 2: tuberin domain}

The tuberin domain of TSC2 protein comprises mainly alpha helices connected by loops in an arrangement similar to alpha-alpha superhelix domain and consists of two distinct subdomains linked through a long helix. The substitutions observed involved residues Lys506 and Glu546 in subdomain 1 and residues Cys644 and Leu717 in subdomain 2.

Five variants were identified in the tuberin domain (p.Lys506Glu, p.Glu546Lys, p.Leu717Pro, p.Cys644Gly, and p.Leu612Pro). Protein modeling was done for Lys506Glu and Glu546Lys present in subdomain 1 and residues Cys644Gly and Leu717Pro in subdomain 2.

In the wild type protein, the positively charged residue Lys506 present in subdomain 1 on the alpha helix forms a salt bridge with negatively charged side chain of Asp567 present on neighbouring helix (Fig. 4a). This is the most significant interaction between these two helices.
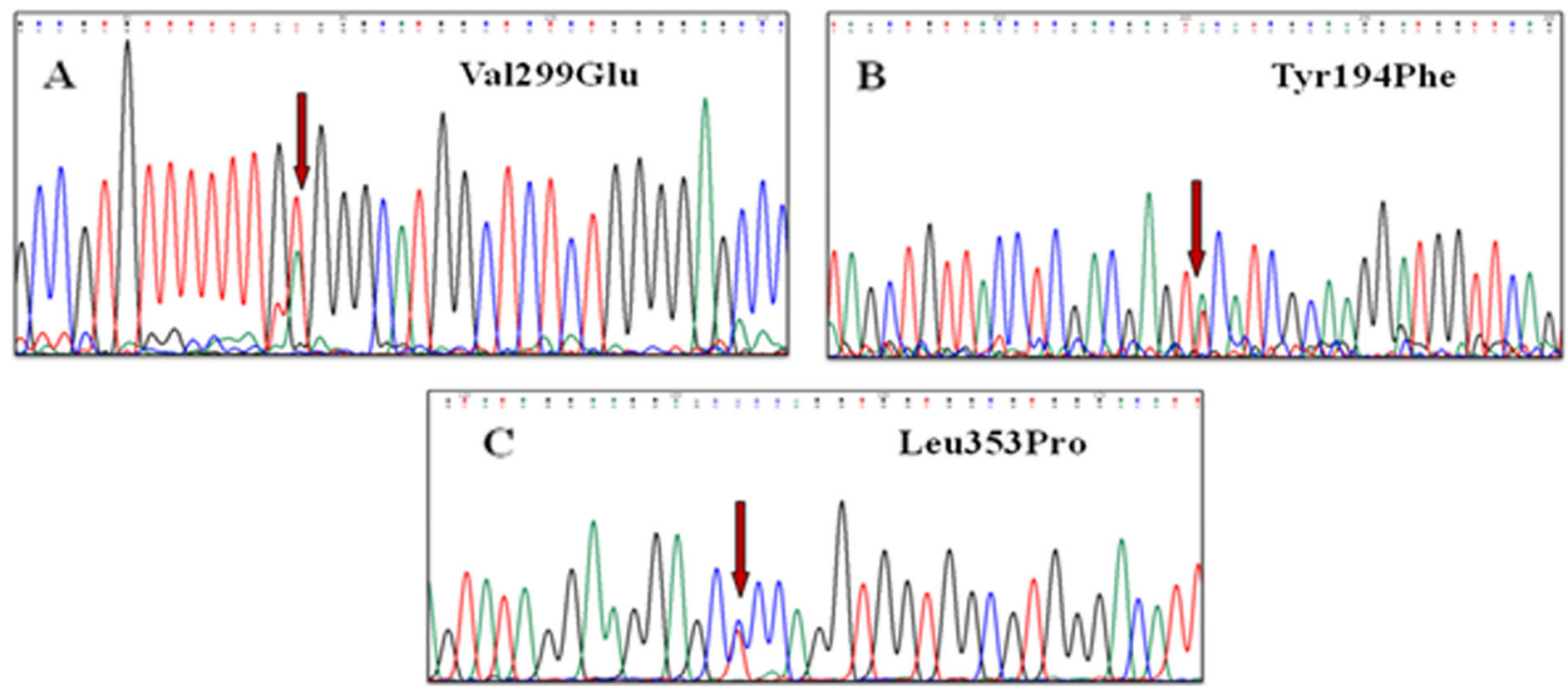

Fig. 2 Electropherogram showing variants identified in Domain 1, (a) Val299Glu (b) Tyr194Phe (c) Leu353Pro, of tuberin protein 


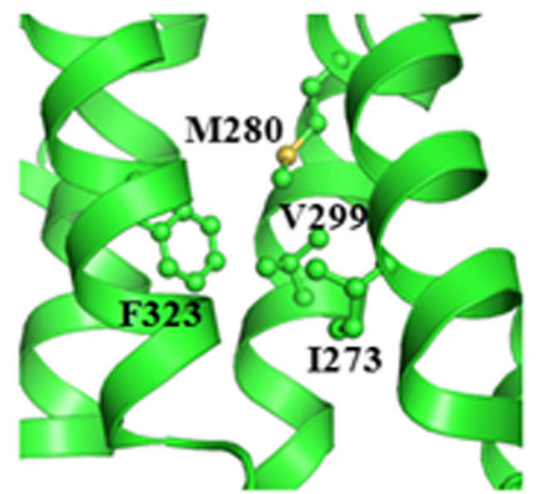

a

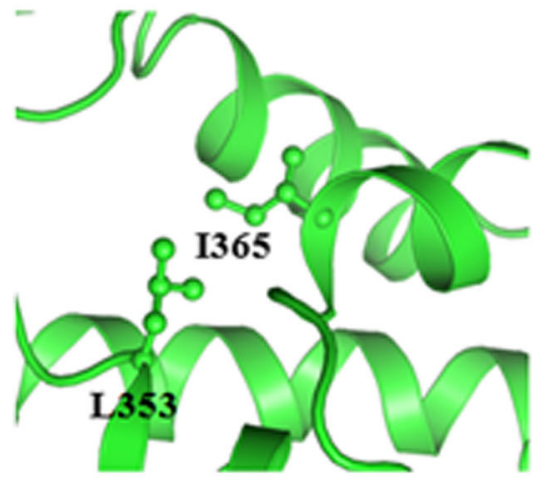

c

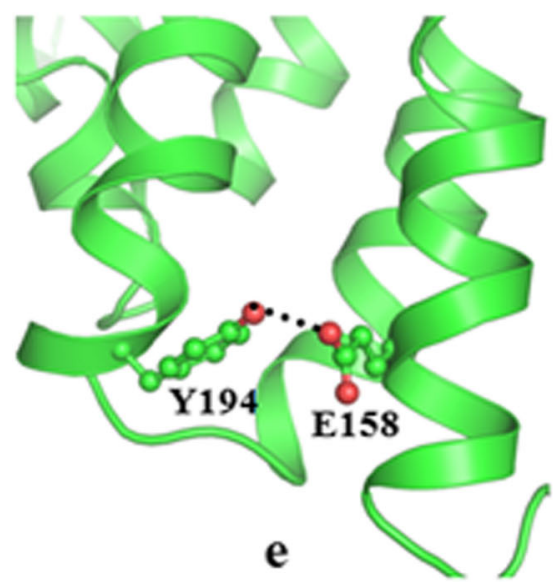

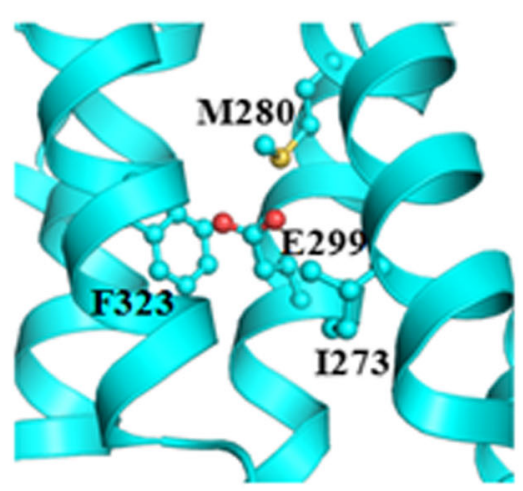

b

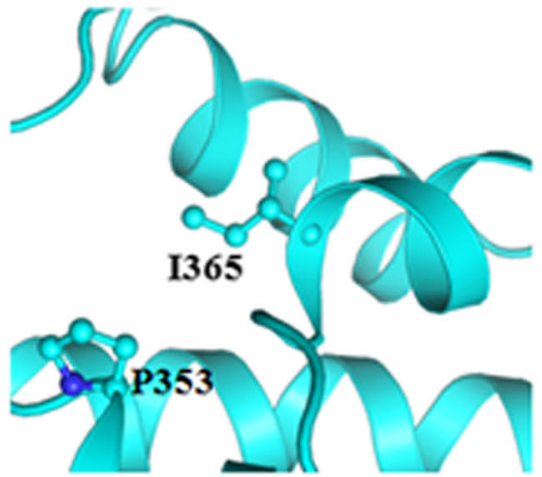

d

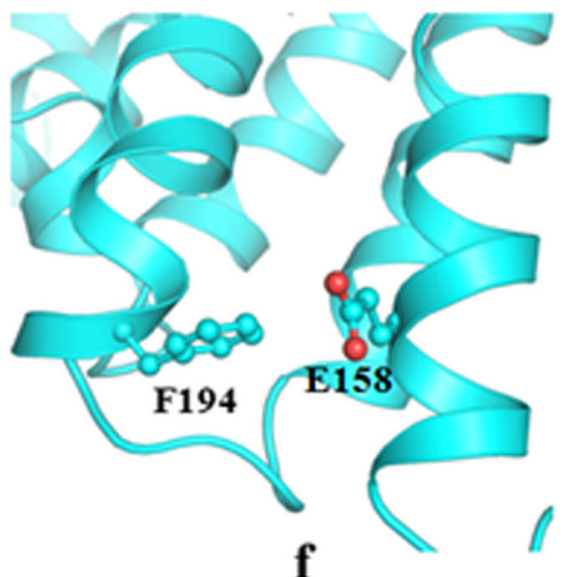

Fig. 3 Cartoon representation of TSC1 interacting domain of wild typeTSC2 (cartoon, green) in (a, c \& e) and mutants (cartoon, cyan) in (b) Val299Glu, (d) Leu353Pro and (f) Tyr194Phe. Important residues have been represented in ball and stick and hydrogen a bonded interaction is shown as black dotted lines

Replacement of positively charged Lys506 in the mutant with the negatively charged Glu506 (Fig. 4) disturbs the charge, environment leading to the loss of the salt bridge and consequently repels the similarly charged side chain of Asp567 (Fig. 4b). This will disturb the interaction between the two helices and adversely impact the stability of the structure.
The presence of the oppositely charged residues, negatively charged Glu546 and positively charged Arg585 on the neighbouring helices results in the formation of a salt bridge which stabilizes the helix-helix interaction (Fig. 4a). The substitution of Glu546 by a positively charged residue Lys546 (Fig. 4) alters the polar environment and pushes the similarly charged 

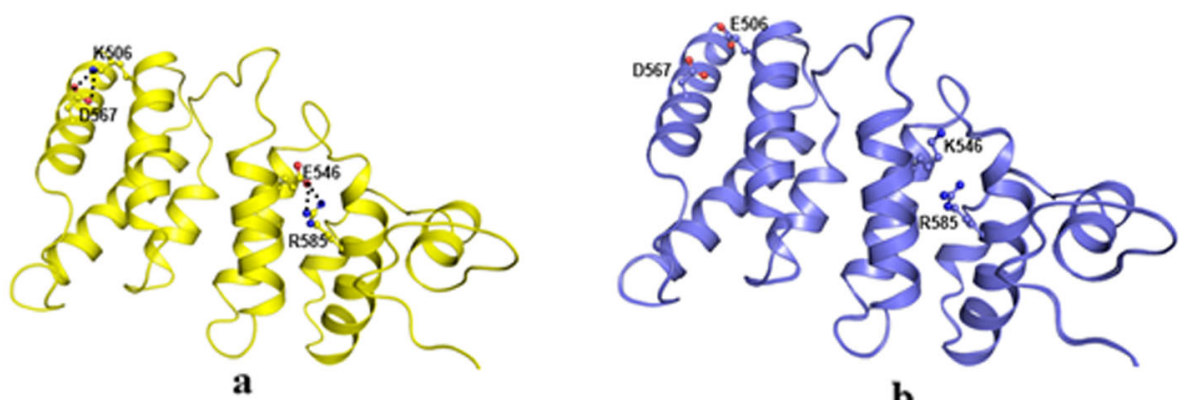

b
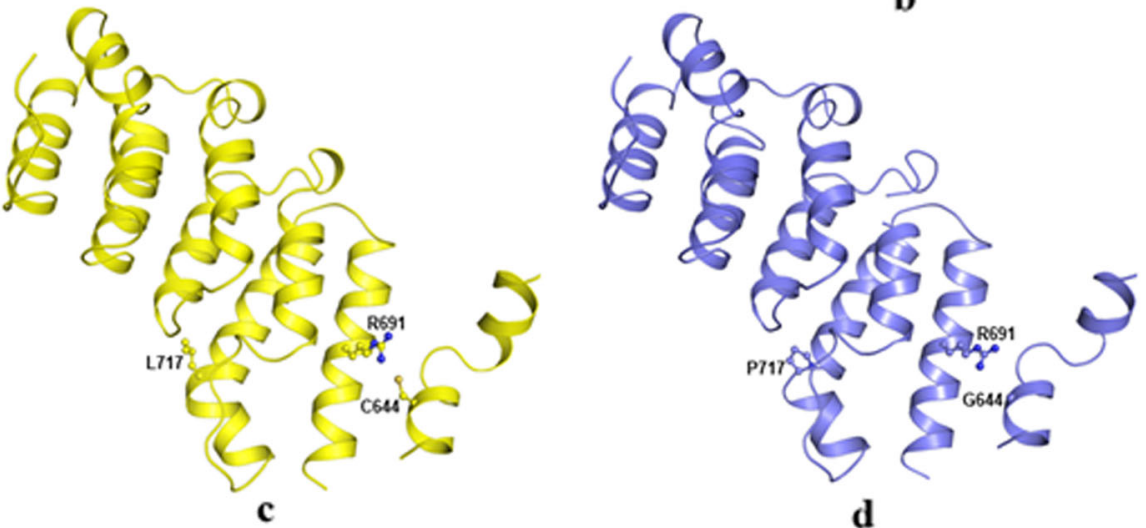

Fig. 4 Cartoon representation of tuberin domain of wild type TSC2 (cartoon, yellow) in (a \& c) and (b) mutants (cartoon, blue) in (b) Lys506Glu and (d) Glu546Lys. Important residues have been represented in ball and stick and hydrogen bonded interactions are shown as black dotted lines

Arg585 away (Fig. 4b). The variant resulting in the loss of salt bridge will adversely impact the helixhelix interaction and might consequently destroy the protein conformation necessary for the dimerization of the protein.

Subdomain 2 consists predominantly of alpha helices similar to the subdomain 1. Residue Cys644 in the wild type protein resides on the linker helix. Due to the absence of an another cysteine residue in the vicinity, it forms a weak hydrogen bond with Arg691 present on the first helix of the second subdomain (Fig. 4c). The mutant has Gly in place of Cys644 which is a supple residue and lacks a side chain. Hence the interaction between subdomain 1 and linker helix is lost in the mutant (Fig. 4d). Another mutant concerning Leu717 residue is located in the long helix in subdomain 2 (Fig. 4c). In the mutant this is replaced by smaller Pro residue. Pro residue contains an imino group and is a well known helix breaker. The replacement of Leu by the Pro in mutant protein unwinds the helix and alters the backbone structure locally (Fig. 4d). This might be a destabilizing factor for the protein.

Clinical assessment The detailed clinical assessment for these 4 TSC patients showed no specific phenotype.

\section{Domain 3: Rap-Gap domain}

The model structure of GAP domain of TSC2 shows the presence of beta sheet consisting of seven parallel beta strands surrounded by alpha helices forming an alphabeta fold. This domain has a catalytic role of GTP hydrolysis. The observed variant Pro1497 in this domain lies in the loop region and connects this domain to another domain of the TSC2 protein (Fig. 6a).

Five variants (p.Pro1497Leu, p.Glu1230Lys, p.Ala1238Val, p.Val1673Ala, and p.His1620Pro) were found in RapGap domain. Protein modeling was done for Pro1497Leu, and Val1673Ala (Fig. 5). The other variants could not be modeled due to the lack of a suitable homology sequence.

The imino group containing Pro residue is also known to provide rigidity in protein structure due to its restricted geometry which could be crucial for the proper arrangement of domains and hence the domain-domain interactions for the functional integrity of TSC2 protein. The substituted residue Leu (Fig. 6) is also a non-polar residue but lacks the rigidity provided by Pro residue. Though Leu1497 can interact with non-polar residue Phe1499 in the same loop through hydrophobic interaction, but it fails to provide the required rigidity and conformation imparted by Pro. The lack of restricted movement (provided by Pro in the wild type) and resultant greater flexibility of the loop due to presence of Leu 


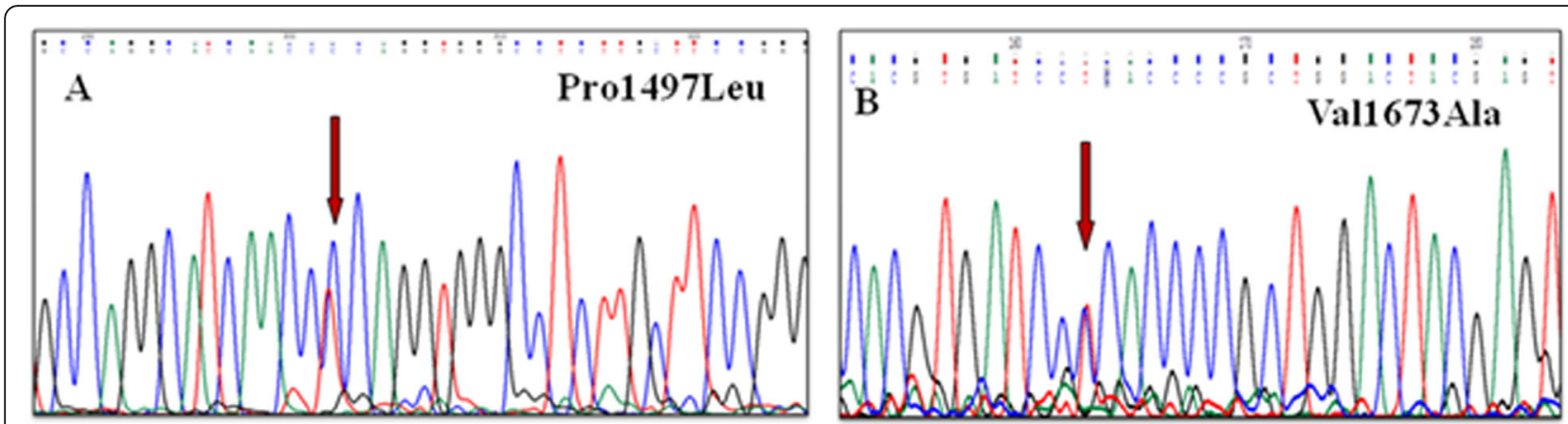

Fig. 5 Electropherogram showing variants identified in Domain 3, (a) Pro1497Leu (b) Val1673Ala, of tuberin protein

(in the mutant) may adversely affect the interaction between the two flanking domains due to possibly greater inter-domain movement resulting in diminished function of the protein.

The Val1673Ala variant might adversely impact the function (Fig. 6). Val1673 is a non-polar residue packed in the hydrophobic pocket surrounded by Phe1574 and Tyr1650. The substituted Ala is smaller in size and found to be less hydrophobic than $\mathrm{Val}$ and this substitution results in the loss of hydrophobic interactions. This may affect the packing and hydrophobic arrangement in the region and hinder GAP activity.

Clinical assessment The detailed clinical assessment for all patients showed presence of seizures and brain lesions. All patients had milder form of neurocognitive outcome as compared to those having variants in domain $1 \& 2$.
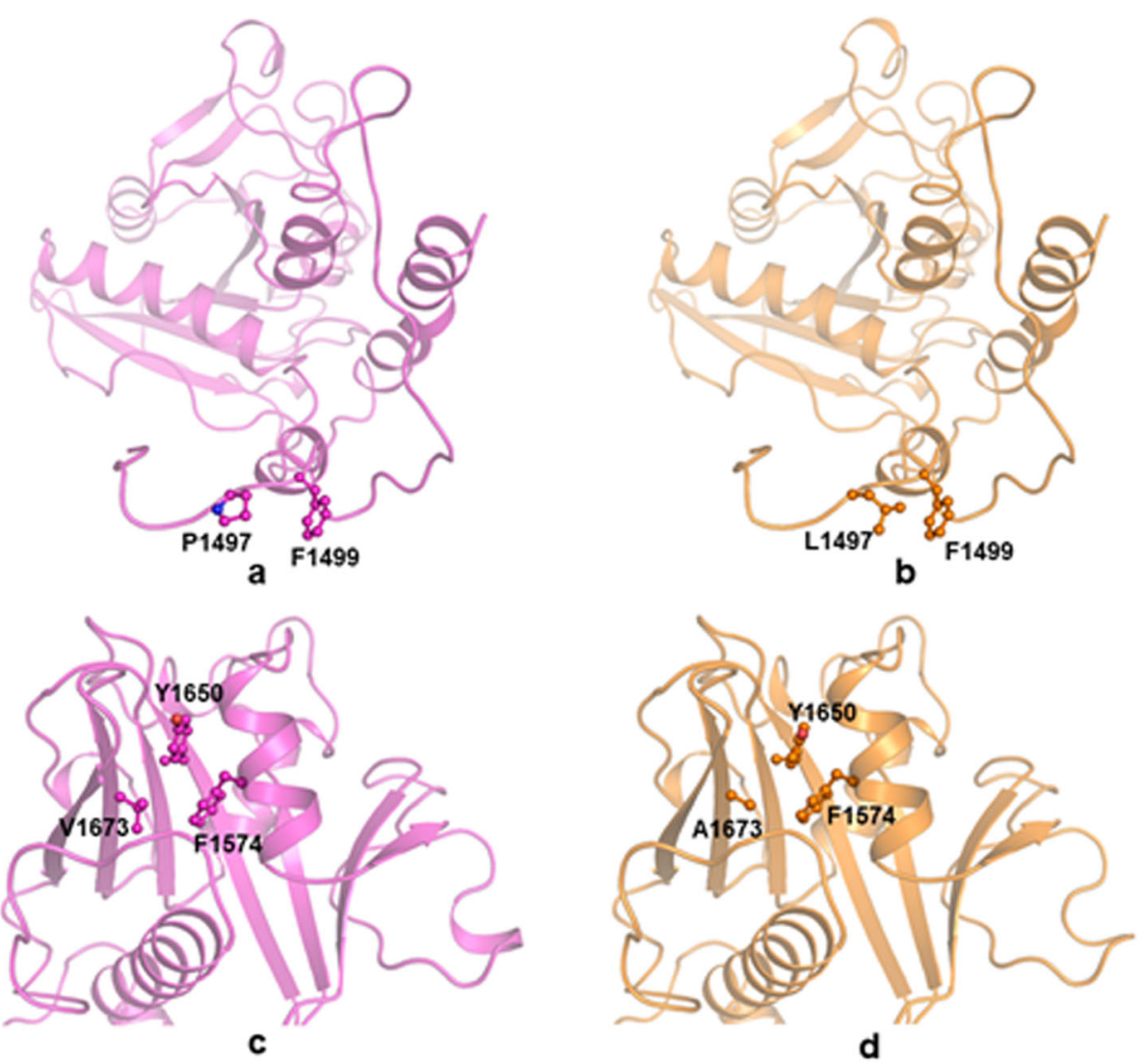

Fig. 6 Cartoon representation of GTPase (GAP) domain of wild type (magenta) TSC2 in (a and c) and mutants (orange) in (b) Pro1497Leu and (d) Val1673Ala. Important residues have been represented in ball and stick 


\section{Discussion}

Studies indicate that the impact of the missense change depends on criteria such as the evolutionary conservation of an amino acid or nucleotide, the location and context within the protein sequence, and the biochemical consequences of the amino acid substitution. As per the study by Thusberg et al. [18] most of the prediction algorithms used for the prediction of missense variants are 65-80\% accurate when examining known disease variants. As per the ACMG standards and guidelines, it recommends that multiple prediction software programs should be used for sequence variant interpretation as each in silico tools have their own weaknesses and strengths.

There were 23 novel variants identified in the present study, of which 14 were missense variants, 3 were inherited variants while remaining 11 were de novo origin as shown in Table 1. These variants were checked in 1000 Genome, dbSNP, HGMD, LOVD and different repository databases of commercial companies like Centogene, MedGenome and Strands and none of these were found in any ethnic population.

Molecular modeling was done for 10 of the total 14 missense variants as for remaining 4 variants no homology protein sequence was identified to generate model structure. The 10 variants were found disrupting the hydrophobic interactions between helices and were also found changing the polarity of the affected domains. This destabilizes the protein structure, thereby affecting the interaction with TSC1. The three dimensional (3D) model of tuberin protein domains was constructed by using a combination of threading server Phyre2 and Discovery studio v2.5 molecular modeling program. The known crystal structure of clathrin adaptor core protein, Rap1-GAP domain and cryo-electron microscopy structure of Ser/Thr kinase Tor protein was used as template respectively. The initial models of both the wild type and mutant type of tuberin protein were subsequently subjected to molecular dynamics (MD) simulations to relax the structure by energy minimization and were verified for stereochemical quality.

The three variants were identified in domain 1 of tuberin protein, which included p.Tyr194Phe, p.Val299Glu and p.Leu353Pro. The substitution at 299 position disrupted the hydrophobic interactions and also changed the polarity of helices from non polar to negatively charged helices. Prolines are known for their rigid structure conformation while at times it forces to change the backbone of protein structure into specific conformation. The substitution at 353 position has disrupted the hydrophobic interactions which weakened the helices and had changed the structure conformation of the protein. Another substitution at 194 position has modified the stability of helices by loss of hydrogen bond interaction thereby decreasing the stability of the domain as shown in Fig. 2.

Similarly in domain 2, among the 5 variants identified protein modeling was done for 4 variants. The substitution at 506 position adversely affected the stability of the structure by disrupting the most significant salt bridge interaction between two helices. Another substitution at 546 by a positively charged residue has changed the polarity of the helices. The substitution at 644 and 717 position weakens the hydrogen bond in helices and the proline in the mutant structure unwinds the helices thereby resulting in disrupting the domain structure. These variants have an adverse impact on the helix-helix interaction and might have destroyed the protein conformation which is necessary for the dimerization of the protein.

The Rap-GAP domain had five variants identified and protein modeling was done for two of them. The substitution at 1497 position disrupted the rigidity of the protein conformation by affecting the stability of the loop in the helical region. Another substitution at 1673 position disrupted the hydrophobic interactions within the helical region which might have affected the packing and hydrophobic arrangement in the region and hindered GAP activity.

Studies from different populations have provided insights about the functional relevance of tuberin and hamartin proteins stating that it might affect cell proliferation, growth, adhesion, migration or protein trafficking [5]. The C- terminal coiled coil domain of hamartin protein is necessary for the interaction with tuberin protein directly to form a complex structure. Also tuberin gets phosphorylated at its serine and tyrosine residues which also affects the interaction between the two proteins to form complex [19]. The GAP domain is responsible for the inhibition of cell division by indirectly modulating the mTOR which is a key regulator of translation [20].

\section{Conclusion}

It is always a challenging task to predict the effect of missense variants on protein function. Analyzing familial segregation could help but the small family size and lack of familial clinical information in simplex cases make the segregation analysis challenging. Considering the functionality of these domains, it is very likely that these missense variants are directly affecting by interfering in protein folding, their charge and hydrophobic interactions leading to protein truncation. To determine the pathogenecity of novel variants many alternative protocols to animal studies are being used nowadays which can provide dependable outcomes. To ascertain whether the 
novel variants identified in TSC patients are disease causing or benign, an attempt was made to understand the level and extent of pathogenecity of these identified novel variants by using different bioinformatics tools and computer aided protein modeling methods.

\section{Abbreviations}

CNS: Central nervous system; RHEB: Ras homolog enriched in brain:

SEGAs: Subependymal giant cell astrocytomas; SEN: Subependymal nodules;

TSC: Tuberous Sclerosis Complex; WML: White matter lesions

\section{Acknowledgements}

We thank all the TSC families for their participation and cooperation and Indian Council of Medical Research (ICMR) for providing financial support to conduct the study. Dr. Manoj Kumar and Dr. Punit Kaur gratefully acknowledge the support by Indian Council of Medical Research (ICMR) through grant of the Bio-Medical Informatics Centre.

\section{Authors' contributions}

SSu- Performed molecular testing and manuscript preparation. MKu and PKPerformed protein modeling and contributed for result section in manuscript preparation. AK- Radiological evaluation. GS- Dermatological evaluation. SG Neurological evaluation. SSa- Developmental assessment. NG- Genetic counselling and clinical evaluation. MKa- Intellectual content, Genetic counselling and clinical evaluation, manuscript final approval. MRC- Concept and design, intellectual content, manuscript preparation and final approval. All authors read and approved the final manuscript.

\section{Funding}

The study was approved and funded by Indian Council of Medical Research (ICMR). There was no role of funding agency in the design of the study and collection, analysis and interpretation of data and in writing manuscript.

\section{Availability of data and materials}

The protein modeling data sets generated during the current study are available from the authors from Department of Biophysics on request. Dr. Punit Kaur: punitkaur1@hotmail.com Dr. Manoj Kumar: manmath. manoj@gmail.com

\section{Ethics approval and consent to participate}

Ref. No. IESC/T-196/03.05.2013, Institutional Sub Ethics committee, All India Institute of Medical Sciences, New Delhi. Written informed consent was obtained from all study participants.

\section{Consent for publication}

Not applicable

\section{Competing interests}

The authors declare that they have no competing interests.

\section{Author details}

'Division of Genetics, Department of Pediatrics, AllMS, New Delhi, India. 2Department of Biophysics, AllMS, New Delhi, India. ${ }^{3}$ Department of Radiodiagnosis, AllMS, New Delhi, India. ${ }^{4}$ Department of Dermatology \& Venerology, AllMS, New Delhi, India. ${ }^{5}$ Division of Neurology, Department of Pediatrics, AllMS, New Delhi, India.

Received: 21 June 2019 Accepted: 9 September 2019 Published online: 26 October 2019

\section{References}

1. Orlova KA, Crino PB. The tuberous sclerosis complex. Ann N Y Acad Sci. 2010;1184:87-105.

2. Tsai V, Crino PB. Tuberous sclerosis complex: genetic basis and management strategies. Adv Genomics Genet. 2012;2:19-31.

3. Caban C, Khan N, Hasbani DM, Crino PB. Genetics of tuberous sclerosis complex: implications for clinical practice. Appl Clin Genet. 2017;10:1-8.
4. Plank TL, Yeung RS, Henske EP. Hamartin, the product of the tuberous sclerosis I (TSC1) gene, interacts with tuberin and appears to be localized to cytoplasmic vesicles. Cancer Res. 1998;58:4766-70.

5. van Slegtenhorst M, Nellist M, Nagelkerken B, Cheadle J, Snell R, van den Ouweland A, Reuser A, Sampson J, Halley D, van der Sluijs P. Interaction between hamartin and tuberin, the TSC1 and TSC2 gene products. Hum Mol Genet. 1998;7(6):1053-7.

6. Castro AF, Rebhun JF, Clark GJ, et al. Rheb binds tuberous sclerosis complex 2 (TSC2) and promotes $\$ 6$ kinase activation in a rapamycin- and farnesylation-dependent manner. J Biol Chem. 2003;278:32493-6.

7. Wullschleger S, Loewith R, Hall MN. TOR signaling in growth and metabolism. Cell. 2006;124:471-84.

8. Kwiatkowska J, Wigowska-Sowinska J, Napierala D, Slomski R, Kwiatkowski D. Mosaicism in tuberous sclerosis as a potential cause of the failure of molecular diagnosis. N Engl J Med. 1999;340(9):703-7.

9. Northrup, Krueger DA, on behalf of the International Tuberous Sclerosis Complex Consensus Group. Tuberous sclerosis complex diagnostic criteria update: recommendations of the 2012 international tuberous sclerosis complex consensus conference. Pediatr Neurol. 2013;49(4):243-54. https:// doi.org/10.1016/j.pediatrneurol.2013.08.001.

10. Sunyaev S, Ramensky V, Koch I, Lathe W 3rd, Kondrashov AS, Bork P. Prediction of deleterious human alleles. Hum Mol Genet. 2001;10(6):591-7.

11. Ng PC, Henikoff S. SIFT: predicting amino acid changes that affect protein function. Nucleic Acids Res. 2003:31(13):3812-4.

12. Schwarz JM, Rödelsperger C, Schuelke M, Seelow D. Mutation Taster evaluates disease-causing potential of sequence alterations. Nat Methods. 2010;7(8):575-6.

13. Kelley LA, Mezulis S, Yates CM, Wass MN, Sternberg MJ. The Phyre2 web portal for protein modeling, prediction and analysis. Nat Protoc. 2015;10(6):845-58.

14. Collins BM, Mccoy AJ, Kent HM, Evans PR, Owen DJ. Molecular architecture and functional model of the endocytic Ap2 complex. Cell. 2002;109:523-35.

15. Daumke O, Weyand M, Chakrabarti PP, Vetter IR, Wittinghofer A. The GTPase activating protein Rap1GAP uses a catalytic asparagine. Nature. 2004:429:197-201.

16. Baretic D, Berndt A, Ohashi Y, Johnson CM, Williams RL. Tor forms a dimer through an $\mathrm{N}$-terminal helical solenoid with a complex topology. Nat Commun. 2016;7:11016.

17. Molecular simulation methods with gromacs. Hands on tutorial. Introduction to molecular dynamics: simulation of lysozyme in water. http:// www.gromacs.org.

18. Thusberg J, Olatubosun A, Vihinen M. Performance of mutation pathogenicity prediction methods on missense variants. Hum Mutat. 2011:32(4):358-68.

19. Aicher LD, Campbell JS, Yeung RS. Tuberin phosphorylation regulates its interaction with hamartin. Two proteins involved in tuberous sclerosis. J Biol Chem. 2001;276(24):21017-21.

20. Wienecke R, Konig A, DeClue JE. Identification of tuberin, the tuberous sclerosis-2 product: Tuberin possesses specific Rap1GAP activity. J Biol Chem. 1995;270(27):16409.

\section{Publisher's Note}

Springer Nature remains neutral with regard to jurisdictional claims in published maps and institutional affiliations.
Ready to submit your research? Choose BMC and benefit from:
- fast, convenient online submission
- thorough peer review by experienced researchers in your field
- rapid publication on acceptance
- support for research data, including large and complex data types
- gold Open Access which fosters wider collaboration and increased citations
- maximum visibility for your research: over $100 \mathrm{M}$ website views per year
At BMC, research is always in progress.
Learn more biomedcentral.com/submission 